\title{
Correction to: The predictive value of body mass index on prognosis and adverse events of cancers treated with immunotherapy: a systematic review and meta-analysis
}

\author{
Yafei You ${ }^{1,2} \cdot$ Chang Jiang $^{1,2} \cdot$ Kunwei Peng $^{1,2} \cdot$ Wenzhuo $\mathrm{He}^{1,2} \cdot$ Lei Wang $^{1,2} \cdot$ Yanan Jin $^{1,2} \cdot$ Liangping Xia $^{1,2} \mathbb{D}$
}

Published online: 20 March 2021

(c) Springer-Verlag GmbH Germany, part of Springer Nature 2021

\section{Correction to: Cancer Immunology, Immunotherapy https://doi.org/10.1007/s00262-021-02858-y}

The original version of this article unfortunately contained a mistake. There is a spelling error in the affiliations (Sun Yat-sen University Cancer Center but not Sun Yat-Senen University Cancer Center).

The correct affiliations should be:

State Key Laboratory of Oncology in South China, Collaborative Innovation Center for Cancer Medicine, Sun YatSen University Cancer Center, 651 Dongfeng East Road, Guangzhou 510060, Guangdong, People's Republic of China.
Department of The VIP Region, Sun Yat-Sen University Cancer Center, Guangzhou 510060, Guangdong, People's Republic of China.

Publisher's Note Springer Nature remains neutral with regard to jurisdictional claims in published maps and institutional affiliations.

The original article can be found online at https://doi.org/10.1007/ s00262-021-02858-y.

Liangping Xia

xialp@sysucc.org.cn

1 State Key Laboratory of Oncology in South China, Collaborative Innovation Center for Cancer Medicine, Sun Yat-Sen University Cancer Center, 651 Dongfeng East Road, Guangzhou 510060, Guangdong, People's Republic of China

2 Department of The VIP Region, Sun Yat-Sen University Cancer Center, Guangzhou 510060, Guangdong,

People's Republic of China 